\title{
Approximation to multifractional Riemann-Liouville Brownian sheet
}

\author{
Hongshuai Dai* \\ School of Mathematics and Information Sciences, Guangxi University, \\ Nanning, 530004 China
}

July 11, 2018

\begin{abstract}
In this paper, we first introduce multifrational Riemann-Liouville Brownian sheets. Then, we show a result of approximation in law of the multifractional RiemannLiouville Brownian sheet. The construction of these approximations is based on a sequence of I.I.D random variables.
\end{abstract}

MSC(2000) 60F17, 60G15.

Keywords: Multifractional Riemann-Liouville Brownian sheet, Donsker's theorem, weak convergence.

\section{Introduction}

A one-dimensional fractional Brownian motion (FBM) $\xi_{H}=\left\{\xi_{H}(t) ; t \in \mathbb{R}_{+}\right\}$with Hurst index $H \in(0,1)$ is a real-valued, centered Gaussian process with covariance function given by

$$
\mathbb{E}\left[\xi_{H}(t) \xi_{H}(s)\right]=\frac{1}{2}\left[|s|^{2 H}+|t|^{2 H}-|t-s|^{2 H}\right], s, t \in \mathbb{R}_{+} .
$$

It was introduced, as a moving-average Gaussian process, by Mandelbrot and Van Ness [12.

Fractional Brownian motion possesses interesting properties such as self-similarity of order $H \in(0,1)$, stationary increments and long-range dependence (when $H>\frac{1}{2}$ ), which make it a good candidate for modeling different phenomena in, for example, finance and telecommunication. However, it does not represent a casual time-invariant system as there is no well-defined impulse response function. Barnes and Allan [2] introduced the fractional Riemann-Liouville (RL) Brownian motion (RL-FBM) $V_{H}=\left\{V_{H}(t) ; t \in \mathbb{R}_{+}\right\}$ based on the following Riemann-Liouville fractional integral,

$$
V_{H}(t)=\frac{1}{\Gamma\left(H+\frac{1}{2}\right)} \int_{\mathbb{R}_{+}}(t-u)_{+}^{H-\frac{1}{2}} d B(u),
$$

\footnotetext{
*E-mail: mathdsh@gmail.com
} 
where $B(u)$ is a standard Brownian motion, $x_{+}=\max \{x, 0\}$, and $\Gamma$ is the gamma function.

$V_{H}$ represents a linear system driven by white noise with the impulse response function $\frac{t^{H-\frac{1}{2}}}{\Gamma\left(H+\frac{1}{2}\right)} \cdot V_{H}$ shares with $\xi_{H}$ many properties which include self-similarity, regularity of sample paths, etc.- with one notable exception that its increment process is non stationary. But it was shown by Lim [14 that the increment process satisfies some weaker forms of stationary. This provides some flexibility necessary for practical applications (see Lim [14] for more information). However, this model may be restrictive, due to the fact that all of its regularity and fractal properties are governed by the single Hurst parameter $H$. In order to overcome this limitation, Lim and Muniandy [16] extended RL-FBM to RLmultifractional Brownian motion (RL-MBM). Roughly speaking, RL-MBM is governed by a Hurst function $H(t)$ with certain regularity in place of the constant Hurst parameter $H$ in RL-FBM. We refer to Lim [14] and Lim and Muniandy [15, 16] for properties of RL-MBMs.

There are two typical multiparameter extensions of FBMs. One is the Lévy's fractional Brownian random field with parameter $H \in(0,1)$ (see Ciesielski and Kamont [6]) : a centered Gaussian process $Y$ with covariance function given by

$$
\mathbb{E}[Y(t) Y(s)]=\frac{1}{2}\left[\|s\|^{2 H}+\|t\|^{2 H}-\|t-s\|^{2 H}\right], s, t \in \mathbb{R}_{+}^{d},
$$

where $\|\cdot\|$ denotes the Euclidean norm.

The other multiparameter extension of the fractional Brownian motion is the anisotropic fractional Brownian sheet introduced by Kamont [11. Since then, fractional Brownian sheets have been studied extensively as a representative of anisotropic Gaussian random fields. See, for example, Wu and Xiao [18] and the references therein for further information. Still, the regularity of fractional Brownian sheets does not evolve in the $d-$ dimensional time parameter $t \in \mathbb{R}_{+}^{d}$.

To model the anisotropic Gaussian random fields whose regularity evolves in time, such as images, Ayache and Léger [1] and Herbin [9] introduced so-called multifractional Brownian sheets (MFBS) in terms of their moving average representations and harmonizable representations, where the constant Hurst vector of fractional Brownian sheets is substituted by Hurst functionals. We refer to Ayahce and Léger [1, Herbin [9], Meerschaert, $\mathrm{Wu}$ and Xiao [13] and the references therein for more information.

Now, we are ready to introduce the multifractional Riemann-Liouville Brownian sheet (RL-MBS).

Definition 1.1 Let $H(t)=\left(H_{1}(t), \cdots, H_{d}(t)\right)$ be a measurable function in $t \in \mathbb{R}_{+}^{d}$ with values in $(0,1)^{d}$. A real-valued RL-multifractional Brownian sheet $X=\{X(t) ; t \in$ $\left.\mathbb{R}_{+}^{d}\right\}$ with functional Hurst index $H(t)$ is defined as the following Riemann-Liouville (RL) fractional integral

$$
X(t)=\int_{\mathbb{R}_{+}^{d}} \prod_{i=1}^{d}\left(t_{i}-u_{i}\right)_{+}^{H_{i}(t)-\frac{1}{2}} W(d u),
$$

where $W(u)=\left\{W(u) ; u \in \mathbb{R}_{+}^{d}\right\}$ is a $d$-parameter Wiener process.

Some authors have studied weak convergence to multifractional Brownian motions. Dai and Li [7] presented a weak limit theorem for the multifractioan Brownian motion based 
on a Poisson process. By using Donsker's theorem, Dai [8] showed an approximation of the RL-multifractional Brownian motion in Besov spaces. On the other hand, Weak limit theorems for fractional Brownian sheets have attracted significant interest in recent years. For example, Bardina et al. 4 gave a result of approximation in law, in the space of the continuous functions on $[0,1]^{2}$, of two-parameter fractional Brownian sheet. Bardina and Florit [3] showed a result of approximation in law of the $d$-parameter fractional Brownian sheet in the space of continuous functions on $[0,1]^{d}$.

Let us recall some known facts. Let $\left\{Z_{k} ; k=\left(k_{1}, k_{2}, \ldots, k_{d}\right) \in \mathbb{N}^{d}\right\}$ be an independent family of centered identically distributed random variables with variance 1 . Wichura [17] generalized Donsker's theorem and proved that the process

$$
n^{\frac{d}{2}} \int_{0}^{t_{d}} \cdots \int_{0}^{t_{1}} \sum_{k \in \mathbb{N}^{d}} Z_{k} I_{[k-1, k)}(u \cdot n) d u
$$

where $I_{[k-1, k)}(u \cdot n)=I_{\left[k_{1}-1, k_{1}\right) \times \cdots \times\left[k_{d}-1, k_{d}\right)}\left(u_{1} n, \cdots, u_{d} n\right)$, converges in law to a $d$ parameter Wiener process.

Inspired by Wichura [17] and Bardina and Florit [3], it is natural to try to approximate $X$ in law by

$$
X_{n}(t)=\int_{\mathbb{R}_{+}^{d}}\left[\prod_{i=1}^{d}\left(t_{i}-u_{i}\right)_{+}^{H_{i}(t)-\frac{1}{2}}\right] \theta_{n}(u) d u
$$

where $\theta_{n}(u)=n^{\frac{d}{2}} \sum_{k \in \mathbb{N}^{d}} Z_{k} I_{[k-1, k)}(u \cdot n)$.

In the rest of this paper, we assume that $H(t)=\left(H_{1}(t), \cdots, H_{d}(t)\right)$ satisfies the following conditions:

- For every $i \in\{1, \cdots, d\}, 0<\alpha_{i} \leq H_{i}(t) \leq \beta_{i}<1$ for all $t \in \mathbb{R}_{+}^{d}$.

- $H(t)$ is $\gamma$-Hölder continuous, i.e.,

$$
\|H(t)-H(s)\| \leq K\|t-s\|^{\gamma}, t, s \in \mathbb{R}_{+}^{d},
$$

where $K>0$ is a constant.

Furthermore, we assume that $\mathbb{E}\left|Z_{k}\right|^{m}<\infty$ for any $k \in \mathbb{N}^{d}$ and $m \in \mathbb{N}$.

In this paper, we consider $[0,1]^{d} \subset \mathbb{R}_{+}^{d}$ with the usual partial order. We will prove that the sequence of laws in $\mathcal{C}\left([0,1]^{d}\right)$ of the processes $\left\{X_{n}(t) ; t \in[0,1]^{d}\right\}$ defined by (1.5) converges weakly to the law of $\left\{X(t) ; t \in[0,1]^{d}\right\}$ given by (1.3).

Most of the estimates of this paper contain unspecified constants. An unspecified positive and finite constant will be denoted by $K$, which may not be the same in each occurrence. Sometimes we shall emphasize the dependence of these constants upon parameters.

We end this section with some properties of RL-multifractional Brownian sheets.

Lemma 1.1 For any $s<t \in[0,1]^{d}$, there exits a constant $K>0$ such that

$$
\mathbb{E}[X(t)-X(s)]^{2} \leq K\|t-s\|^{2 H},
$$

where $H=\min _{i \in\{1, \cdots, d\}}\left\{\alpha_{i}, \gamma\right\}$. 
Proof: First, we have

$$
\mathbb{E}[X(t) X(s)]=\Pi_{i=1}^{d}\left[\int_{[0,1]}\left(t_{i}-u_{i}\right)_{+}^{H_{i}(t)-\frac{1}{2}}\left(s_{i}-u_{i}\right)_{+}^{H_{i}(s)-\frac{1}{2}} d u_{i}\right] .
$$

By (1.6), we can consider the process $X=\{X(t)\}$ defined from $d$ independent RLmultifractional Brownian motions $Y^{(i)}$ with parameter $\tilde{H}_{i}\left(t_{i}\right)=H_{i}(t)$ by

$$
Y(t)=Y^{(1)}\left(t_{1}\right) \cdots Y^{(d)}\left(t_{d}\right)
$$

It is easy to see that it has the same covariance function as the RL-mutifractional Brownian sheet $X$.

From above arguments, we get

$$
\begin{aligned}
\mathbb{E}[X(t)-X(s)]^{2}=\mathbb{E}\left[\Pi_{i=1}^{d} Y^{(i)}\left(t_{i}\right)-\Pi_{i=1}^{d} Y^{(i)}\left(s_{i}\right)\right]^{2} \\
=\mathbb{E}\left[\left(\Pi_{i=1}^{d} Y^{(i)}\left(t_{i}\right)-Y^{(1)}\left(s_{i}\right) \Pi_{i=2}^{d} Y^{(i)}\left(t_{i}\right)\right)+\cdots\right. \\
\left.+\left(\Pi_{i=1}^{d-1} Y^{(i)}\left(s_{i}\right) Y^{(d)}\left(t_{d}\right)-\Pi_{i=1}^{d} Y^{(i)}\left(s_{i}\right)\right)\right]^{2} .
\end{aligned}
$$

Since $Y^{(i)}, i=1, \cdots, d$ are mutually independent and $\mathbb{E}\left[Y^{(i)}\right]^{2}<K$, we get that

$$
\mathbb{E}[X(t)-X(s)]^{2} \leq K \sum_{i=1}^{d} \mathbb{E}\left[Y^{(i)}\left(t_{i}\right)-Y^{(i)}\left(s_{i}\right)\right]^{2}
$$

It follows from Dai [8] that

$$
\mathbb{E}\left[Y^{(i)}\left(t_{i}\right)-Y^{(i)}\left(s_{i}\right)\right]^{2} \leq K\left|t_{i}-s_{i}\right|^{2 H_{i}(t)}+K\left|H_{i}(t)-H_{i}(s)\right|^{2} .
$$

Since $t, s \in[0,1]^{d}$,

$$
\left|t_{i}-s_{i}\right| \leq 1
$$

On the other hand, we have

$$
\left|H_{i}(t)-H_{i}(s)\right|<1
$$

By (??) to (1.12), one can easily get that the lemma holds.

It follows from the Kolmogorov continuity theorem (see Theorem 2.3.1 in Khoshnevisan [10]) and Lemma 1.1 that

Corollary 1.1 $\{X(t)\}$ has a continuous modification.

The proof of Corollary 1.1 is classical, so we omit the proof. 


\section{Main result}

The main result of this paper is the following theorem.

Theorem 2.1 The sequence of laws in $\mathcal{C}\left([0,1]^{d}\right)$ of the processes $\left\{X_{n}(t) ; t \in[0,1]^{d}\right\}$ defined by (1.5) converges weakly to the law of $\left\{X(t) ; t \in[0,1]^{d}\right\}$ given by (1.3).

In order to prove Theorem 2.1, we have to check that the family of laws of the processes $\left\{X_{n}\right\}$ is tight. First, we need a technical lemma.

Lemma 2.1 For any even $m \in \mathbb{N}$, there exists a constant $K>0$ such that for any nonnegative functions $f_{i} \in L^{2}([0,1]), i=1, \cdots, d$,

$$
\mathbb{E}\left[\int_{[0,1]^{d}}\left[\prod_{i=1}^{d} f_{i}\left(u_{i}\right)\right] \theta_{n}(u) d u\right]^{m} \leq K \prod_{i=1}^{d}\left[\int_{0}^{1} f_{i}^{2}\left(u_{i}\right) d u_{i}\right]^{\frac{m}{2}} .
$$

Proof: We have

$$
\begin{aligned}
\mathbb{E}\left[\int_{[0,1]^{d}}\left[\prod_{i=1}^{d} f_{i}\left(u_{i}\right)\right] \theta_{n}(u) d u\right]^{m} & =\mathbb{E}\left[\int_{[0,1]^{d m}}\left[\prod_{j=1}^{m} \prod_{i=1}^{d} f_{i}\left(u_{i}^{j}\right)\right]\left[\prod_{j=1}^{m} \theta_{n}\left(u^{j}\right)\right] d u^{1} \cdots d u^{m}\right] \\
& =\int_{[0,1]^{d m}}\left[\prod_{j=1}^{m} \prod_{i=1}^{d} f_{i}\left(u_{i}^{j}\right)\right] \mathbb{E}\left[\prod_{j=1}^{m} \theta_{n}\left(u^{j}\right)\right] d u^{1} \cdots d u^{m}(2.2)
\end{aligned}
$$

On the other hand, we have

$$
\begin{aligned}
\mathbb{E}\left[\prod_{j=1}^{m} \theta_{n}\left(u^{j}\right)\right] & =n^{\frac{d m}{2}} \mathbb{E}\left[\prod_{j=1}^{m}\left(\sum_{k \in \mathbb{N}^{d}} Z_{k} I_{[k-1, k)}\left(u^{j} \cdot n\right)\right)\right] \\
& =n^{\frac{d m}{2}} \mathbb{E}\left[\sum_{k^{1}, \cdots, k^{m} \in \mathbb{N}^{d}} Z_{k^{1}} \cdots Z_{k^{m}} I_{\left[k^{1}-1, k^{1}\right)}\left(u^{1} \cdot n\right) \cdots I_{\left[k^{m}-1, k^{m}\right)}\left(u^{m} \cdot n\right)\right] \\
& \leq K n^{\frac{d m}{2}} \sum_{\left(k^{1}, \cdots, k^{m}\right) \in A_{m}} I_{\left[k^{1}-1, k^{1}\right)}\left(u^{1} \cdot n\right) \cdots I_{\left[k^{m}-1, k^{m}\right)}\left(u^{m} \cdot n\right)
\end{aligned}
$$

where

$$
\begin{aligned}
& A_{m}=\left\{\left(k^{1}, \cdots, k^{m}\right) \in \mathbb{N}^{d m} ; \text { for all } i \in\{1, \cdots, m\},\right. \\
& \text { there exists } \left.j \in\{1, \cdots, m\} \backslash\{i\} \text { such that } k^{i}=k^{j}\right\},
\end{aligned}
$$

since $Z_{k}, k \in \mathbb{N}^{d}$ are independent and $\mathbb{E}\left|Z_{k}\right|^{m}<\infty$ for all $m \in \mathbb{N}$ and $k \in \mathbb{N}^{d}$.

We have

$$
\sum_{\left(k^{1}, \cdots, k^{m}\right) \in A_{m}} I_{\left[k^{1}-1, k^{1}\right)}\left(u^{1} \cdot n\right) \cdots I_{\left[k^{m}-1, k^{m}\right)}\left(u^{m} \cdot n\right) \leq I_{D_{m}}\left(u^{1}, \cdots, u^{m}\right),
$$

where

$D_{m}=\left\{\left(u^{1}, \cdots, u^{m}\right) \in[0,1]^{d m} ;\right.$ for all $i \in\{1, \cdots, m\}$

there exists $j \in\{1, \cdots, m\} \backslash\{i\}$ such that for all $\left.k=1, \cdots, d,\left|u_{k}^{i}-u_{k}^{j}\right| \leq \frac{1}{n}\right\}$. 
In fact, we can bound $I_{D_{m}}$ by a finite sum of products of indictators. Moreover all the $d m$ variables, $u_{1}^{1}, \cdots, u_{d}^{m}$, appear in each product of indicators, but each variable appears only in each one of indicators of each product. Then we can bound (2.2) by a sum of products of the following two kinds of terms:

- For any $i \neq j, i, j \in\{1, \cdots, m\}$,

$$
\tilde{I}_{1}=K n^{d} \int_{[0,1]^{2 d}} \prod_{k=1}^{d}\left[f_{k}\left(u_{k}^{i}\right) f_{k}\left(u_{k}^{j}\right)\right] \prod_{k=1}^{d} I_{\left[0, \frac{1}{n}\right)}\left(\left|u_{k}^{i}-u_{k}^{j}\right|\right) d u^{i} d u^{j} .
$$

- For any $i \neq j \neq r, i, j, r \in\{1, \cdots, m\}$,

$$
\begin{aligned}
\tilde{I}_{2}=K n^{\frac{3 d}{2}} \int_{[0,1]^{3 d}} \prod_{k=1}^{d}\left[f_{k}\left(u_{k}^{i}\right) f_{k}\left(u_{k}^{j}\right) f\left(u_{k}^{r}\right)\right] \\
\\
\prod_{k=1}^{d}\left[I_{\left[0, \frac{1}{n}\right)}\left(\left|u_{k}^{i}-u_{k}^{j}\right|\right) I_{\left[0, \frac{1}{n}\right)}\left(\left|u_{k}^{i}-u_{k}^{r}\right|\right) I_{\left[0, \frac{1}{n}\right)}\left(\left|u_{k}^{j}-u_{k}^{r}\right|\right)\right] d u^{i} d u^{r} d u^{j} .
\end{aligned}
$$

In order to prove the lemma, we only need to show

$$
\tilde{I}_{1} \leq K \int_{[0,1]^{d}} \prod_{k=1}^{d}\left[f_{k}^{2}\left(u_{k}\right)\right] d u
$$

and

$$
\tilde{I}_{2} \leq K\left[\int_{[0,1]^{d}} \prod_{k=1}^{d}\left[f_{k}^{2}\left(u_{k}\right)\right] d u\right]^{\frac{3}{2}} .
$$

First, we show that (2.7) holds. $\tilde{I}_{1}$ can be bounded by

$$
\begin{aligned}
& K n^{d} \int_{[0,1]^{2 d}} \prod_{k=1}^{d}\left[f_{k}\left(u_{k}^{j}\right)\right]^{2} \prod_{k=1}^{d} I_{\left[0, \frac{1}{n}\right)}\left(\left|u_{k}^{j}-u_{k}^{i}\right|\right) d u^{j} d u^{i} \\
& \quad+K n^{d} \int_{[0,1]^{2 d}} \prod_{k=1}^{d}\left[f_{k}\left(u_{k}^{i}\right)\right]^{2} \prod_{k=1}^{d} I_{\left[0, \frac{1}{n}\right)}\left(\left|u_{k}^{j}-u_{k}^{i}\right|\right) d u^{j} d u^{i} .
\end{aligned}
$$

where we have used the elementary inequality $2 a b \leq a^{2}+b^{2}$.

The two terms in (2.9) can be done with the same method. So we only deal with the first one. In fact,

$$
\begin{gathered}
K n^{d} \int_{[0,1]^{2 d}} \prod_{k=1}^{d}\left[f_{k}\left(u_{k}^{j}\right)\right]^{2} \prod_{k=1}^{d} I_{\left[0, \frac{1}{n}\right)}\left(\left|u_{k}^{j}-u_{k}^{i}\right|\right) d u^{i} d u^{j} \\
\leq K \int_{[0,1]^{d}} \prod_{k=1}^{d}\left[f_{k}\left(u_{k}^{j}\right)\right]^{2} d u^{j} .
\end{gathered}
$$

From (2.9) and (2.10), we can get that (2.7) holds. 
Now, we prove (2.8). $\tilde{I}_{2}$ equals

$$
\begin{aligned}
& K n^{\frac{3 d}{2}} \int_{[0,1]^{2 d}}\left[\prod_{k=1}^{d}\left[f_{k}\left(u_{k}^{i}\right) I_{\left[0, \frac{1}{n}\right)}\left(\left|u_{k}^{i}-u_{k}^{j}\right|\right)\right]\right] \\
& {\left[\int_{[0,1] d} \prod_{k=1}^{d}\left[f_{k}\left(u_{k}^{r}\right) f_{k}\left(u_{k}^{j}\right) I_{\left[0, \frac{1}{n}\right)}\left(\left|u_{k}^{i}-u_{k}^{r}\right|\right) I_{\left[0, \frac{1}{n}\right)}\left(\left|u_{k}^{j}-u_{k}^{r}\right|\right)\right] d u^{r}\right] d u^{i} d u^{j} . }
\end{aligned}
$$

By the Hölder inequality,

$$
\tilde{I}_{2} \leq K n^{\frac{3 d}{2}} G_{1}^{\frac{1}{2}} \times G_{2}^{\frac{1}{2}}
$$

where

$$
G_{1}=\int_{[0,1]^{2 d}} \prod_{k=1}^{d}\left[f_{k}\left(u_{k}^{i}\right) I_{\left[0, \frac{1}{n}\right)}\left(\left|u_{k}^{i}-u_{k}^{j}\right|\right)\right]^{2} d u^{i} d u^{j}
$$

and

$$
G_{2}=\int_{[0,1]^{2 d}}\left[\int_{[0,1]^{d}} \prod_{k=1}^{d}\left[f_{k}\left(u_{k}^{r}\right) f_{k}\left(u_{k}^{j}\right) I_{\left[0, \frac{1}{n}\right)}\left(\left|u_{k}^{i}-u_{k}^{r}\right|\right) I_{\left[0, \frac{1}{n}\right)}\left(\left|u_{k}^{j}-u_{k}^{r}\right|\right)\right] d u^{r}\right]^{2} d u^{i} d u^{j} .
$$

Using the same method as the proof of (2.7), we can get

$$
G_{1} \leq K n^{-d} \int_{[0,1]^{d}} \prod_{k=1}^{d} f_{k}^{2}\left(u_{k}\right) d u
$$

Now, we deal with $G_{2} . G_{2}$ equals

$$
\begin{gathered}
\int_{[0,1]^{4 d}} \prod_{k=1}^{d}\left[f_{k}^{2}\left(u_{k}^{j}\right) f_{k}\left(u_{k}^{r_{1}}\right) f_{k}\left(u_{k}^{r_{2}}\right) I_{\left[0, \frac{1}{n}\right)}\left(\left|u_{k}^{r_{1}}-u_{k}^{j}\right|\right) I_{\left[0, \frac{1}{n}\right)}\left(\left|u_{k}^{r_{2}}-u_{k}^{j}\right|\right)\right. \\
\left.I_{\left[0, \frac{1}{n}\right)}\left(\left|u_{k}^{r_{1}}-u_{k}^{i}\right|\right) I_{\left[0, \frac{1}{n}\right)}\left(\left|u_{k}^{r_{2}}-u_{k}^{i}\right|\right)\right] d u^{r_{1}} d u^{r_{2}} d u^{j} d u^{i}
\end{gathered}
$$

So, $G_{2}$ can be bounded by

$$
\begin{aligned}
K n^{-d} \int_{[0,1]^{3 d}} & \prod_{k=1}^{d}\left[f_{k}^{2}\left(u_{k}^{j}\right) f_{k}\left(u_{k}^{r_{1}}\right) f_{k}\left(u_{k}^{r_{2}}\right) I_{\left[0, \frac{1}{n}\right)}\left(\left|u_{k}^{r_{1}}-u_{k}^{j}\right|\right) I_{\left[0, \frac{1}{n}\right)}\left(\left|u_{k}^{r_{2}}-u_{k}^{j}\right|\right)\right] d u^{r_{1}} d u^{r_{2}} d u^{j} \\
& \leq K n^{-d}\left(G_{3}+G_{4}\right),
\end{aligned}
$$

where

$$
G_{3}=\int_{[0,1]^{3 d}} \prod_{k=1}^{d}\left[f_{k}^{2}\left(u_{k}^{j}\right) f_{k}^{2}\left(u_{k}^{r_{1}}\right) I_{\left[0, \frac{1}{n}\right)}\left(\left|u_{k}^{r_{2}}-u_{k}^{j}\right|\right)\right] d u^{r_{1}} d u^{r_{2}} d u^{j}
$$


and

$$
G_{4}=\int_{[0,1]^{3 d}} \prod_{k=1}^{d}\left[f_{k}^{2}\left(u_{k}^{j}\right) f_{k}^{2}\left(u_{k}^{r_{2}}\right) I_{\left[0, \frac{1}{n}\right)}\left(\left|u_{k}^{r_{1}}-u_{k}^{j}\right|\right)\right] d u^{r_{1}} d u^{r_{2}} d u^{j}
$$

$G_{3}$ can be bounded by

$$
\begin{aligned}
\int_{[0,1]^{2 d}} & {\left[\prod_{k=1}^{d} f_{k}^{2}\left(u_{k}^{j}\right) f_{k}^{2}\left(u_{k}^{r_{1}}\right)\right]\left[\int_{[0,1]^{d}} \prod_{k=1}^{d} I_{\left[0, \frac{1}{n}\right)}\left(\left|u_{k}^{r_{2}}-u_{k}^{j}\right|\right) d u^{r_{2}}\right] d u^{r_{1}} d u^{j} } \\
\leq & K n^{-d} \int_{[0,1]^{2 d}} \prod_{k=1}^{d}\left[f_{k}^{2}\left(u_{k}^{j}\right) f_{k}^{2}\left(u_{k}^{r_{1}}\right)\right] d u^{r_{1}} d u^{j} \\
& =K n^{-d}\left[\int_{[0,1]^{d}} \prod_{k=1}^{d}\left[f_{k}^{2}\left(u_{k}\right)\right] d u\right]^{2} .
\end{aligned}
$$

Using the same method as above, we can get that $G_{4}$ can be bounded by

$$
K n^{-d}\left[\int_{[0,1]^{d}} \prod_{k=1}^{d}\left[f_{k}^{2}\left(u_{k}\right)\right] d u\right]^{2} .
$$

It follows from (2.11) to (2.16) that the inequality (2.8) holds. This completes the proof.

Lemma 2.2 For any even $m \in \mathbb{N}$, there exists a constant $K>0$ such that for any $s, t \in[0,1]^{d}$ with $s<t$,

$$
\mathbb{E}\left[X_{n}(t)-X_{n}(s)\right]^{m} \leq K\|t-s\|^{m H}
$$

where $H=\min _{i \in\{1, \cdots, d\}}\left\{\alpha_{i}, \gamma\right\}$.

Proof: It follows from Lemma 2.1 that

$$
\begin{aligned}
\mathbb{E}\left[X_{n}(t)-X_{n}(s)\right]^{m} & \leq K\left[\int_{[0,1]^{d}} \prod_{i=1}^{d}\left(\left(t_{i}-u_{i}\right)_{+}^{H_{i}(t)-\frac{1}{2}}-\left(s_{i}-u_{i}\right)_{+}^{H_{i}(s)-\frac{1}{2}}\right)^{2} d u\right]^{\frac{m}{2}} \\
& \leq K\|t-s\|^{m H} .
\end{aligned}
$$

On the other hand, one can easily prove that

Corollary 2.1 The process $X_{n}$ is continuous for every $t \in[0,1]^{d}$ a.s.

Next, we will prove the main result of this paper.

Proof of Theorem [2.1: By Bickel and Wichura [5], in order to prove Theorem 2.1, we only need to prove that the family of laws of processes $\left\{X_{n}(t)\right\}$ is tight, and identify the limit law of any convergent subsequence as the law of the $d$-parameter RL-multifractional Brownian sheet $X$. The tightness comes from Lemma 2.2 and Bickel and Wichura [5]. 
Now, we proceed to identify the limit law. It is sufficient to show that for any $q \in$ $\mathbb{N}, a_{1}, \cdots, a_{q} \in \mathbb{R}$ and $t^{1}, \cdots, t^{q} \in[0,1]^{d}$,

$$
\mathbb{E}\left[\exp \left\{i \xi \sum_{j=1}^{q} a_{j} X_{n}\left(t^{j}\right)\right\}\right] \rightarrow \mathbb{E}\left[\exp \left\{i \xi \sum_{j=1}^{q} a_{j} X\left(t^{j}\right)\right\}\right]
$$

as $n \rightarrow \infty$.

For any $j \in\{1, \cdots, q\}$ and $i \in\{1, \cdots, d\}$, we have

$$
\left(t_{i}^{j}-u_{i}\right)_{+}^{H_{i}\left(t^{j}\right)-\frac{1}{2}} \in L^{2}([0,1])
$$

and

$$
\left(t_{i}^{j}-u_{i}\right)_{+}^{H_{i}\left(t^{j}\right)-\frac{1}{2}} \geq 0
$$

Therefore, for any $j \in\{1, \cdots, q\}$ and $i \in\{1, \cdots, d\}$, we can find a sequence $\left\{\rho_{i, k}^{j}\left(u_{i}\right)\right\}_{k \in \mathbb{N}}$ of simple functions such that

$$
\int_{[0,1]}\left(\rho_{i, k}^{j}\left(u_{i}\right)-\left(t_{i}^{j}-u_{i}\right)_{+}^{H_{i}\left(t^{j}\right)-\frac{1}{2}}\right)^{2} d u_{i} \rightarrow 0, \text { as } k \rightarrow \infty
$$

and

$$
\left(t_{i}^{j}-u_{i}\right)_{+}^{H_{i}\left(t^{j}\right)-\frac{1}{2}}-\rho_{i, k}^{j}\left(u_{i}\right) \geq 0, \text { for all } k \in \mathbb{N} .
$$

In order to simplify the notation, we define

$$
X_{n}^{j, k}=\int_{[0,1]^{d}}\left[\prod_{i=1}^{d} \rho_{i, k}^{j}\left(u_{i}\right)\right] \theta_{n}(u) d u
$$

and

$$
X^{j, k}=\int_{[0,1]^{d}} \prod_{i=1}^{d} \rho_{i, k}^{j}\left(u_{i}\right) W(d u)
$$

Note that

$$
\left|\mathbb{E}\left[\exp \left\{i \xi \sum_{j=1}^{q} a_{j} X_{n}\left(t^{j}\right)\right\}\right]-\mathbb{E}\left[\exp \left\{i \xi \sum_{j=1}^{q} a_{j} X\left(t^{j}\right)\right\}\right]\right| \leq I_{1}+I_{2}+I_{3}
$$

where

$$
\begin{aligned}
& I_{1}=\left|\mathbb{E}\left[\exp \left\{i \xi \sum_{j=1}^{q} a_{j} X_{n}\left(t^{j}\right)\right\}-\exp \left\{i \xi \sum_{j=1}^{q} a_{j} X_{n}^{j, k}\right\}\right]\right| \\
& I_{2}=\left|\mathbb{E}\left[\exp \left\{i \xi \sum_{j=1}^{q} a_{j} X\left(t^{j}\right)\right\}-\exp \left\{i \xi \sum_{j=1}^{q} a_{j} X^{j, k}\right\}\right]\right|
\end{aligned}
$$


and

$$
I_{3}=\left|\mathbb{E}\left[\exp \left\{i \xi \sum_{j=1}^{q} a_{j} X_{n}^{j, k}\right\}-\exp \left\{i \xi \sum_{j=1}^{q} a_{j} X^{j, k}\right\}\right]\right|
$$

We first deal with $I_{1}$. By the mean value theorem,

$$
I_{1} \leq K \max _{1 \leq j \leq q}\left\{\mathbb{E}\left|X_{n}\left(t^{j}\right)-X_{n}^{j, k}\right|\right\}
$$

On the other hand, for $j \in\{1,2, \cdots, q\}$

$$
\begin{aligned}
& \mathbb{E}\left|X_{n}\left(t^{j}\right)-X_{n}^{j, k}\right| \\
& =\mathbb{E}\left[\mid \int_{[0,1]^{d}}\left[\left(t_{1}^{j}-u_{1}\right)_{+}^{H_{1}\left(t^{j}\right)-\frac{1}{2}}-\rho_{1, k}^{j}\left(u_{1}\right)\right]\left[\prod_{i=2}^{d}\left(t_{i}^{j}-u_{i}\right)_{+}^{H_{i}\left(t^{j}\right)-\frac{1}{2}}\right] \theta_{n}(u) d u d u\right. \\
& \left.\quad+\int_{[0,1]^{d}} \rho_{1, k}^{j}\left(u_{1}\right)\left[\prod_{i=2}^{d}\left(t_{i}^{j}-u_{i}\right)_{+}^{H_{i}\left(t^{j}\right)-\frac{1}{2}}-\prod_{i=2}^{d} \rho_{i, k}^{j}\left(u_{i}\right)\right] \theta_{n}(u) d u \mid\right] \\
& \leq \mathbb{E}\left[\left|\int_{[0,1]^{d}}\left[\left(t_{1}^{j}-u_{1}\right)_{+}^{H_{1}\left(t^{j}\right)-\frac{1}{2}}-\rho_{1, k}^{j}\left(u_{1}\right)\right]\left[\prod_{i=2}^{d}\left(t_{i}^{j}-u_{i}\right)_{+}^{H_{i}\left(t^{j}\right)-\frac{1}{2}}\right] \theta_{n}(u) d u\right|\right] \\
& \quad+\mathbb{E}\left[\left|\int_{[0,1]^{d}} \rho_{1, k}^{j}\left(u_{1}\right)\left[\prod_{i=2}^{d}\left(t_{i}^{j}-u_{i}\right)_{+}^{H_{i}\left(t^{j}\right)-\frac{1}{2}}-\prod_{i=2}^{d} \rho_{i, k}^{j}\left(u_{i}\right)\right] \theta_{n}(u) d u\right|\right] .
\end{aligned}
$$

On the other hand,

$$
\begin{gathered}
\mathbb{E}\left[\left|\int_{[0,1]^{d}}\left[\left(t_{1}^{j}-u_{1}\right)_{+}^{H_{1}\left(t^{j}\right)-\frac{1}{2}}-\rho_{1, k}^{j}\left(u_{1}\right)\right]\left[\prod_{i=2}^{d}\left(t_{i}^{j}-u_{i}\right)_{+}^{H_{i}\left(t^{j}\right)-\frac{1}{2}}\right] \theta_{n}(u) d u\right|\right] \\
\leq K\left[\int_{[0,1]^{d}}\left[\left(\left(t_{1}^{j}-u_{1}\right)_{+}^{H_{1}\left(t^{j}\right)-\frac{1}{2}}-\rho_{1, k}^{j}\left(u_{1}\right)\right) \prod_{i=2}^{d}\left(t_{i}^{j}-u_{i}\right)_{+}^{H_{i}\left(t^{j}\right)-\frac{1}{2}}\right]^{2} d u\right]^{\frac{1}{2}} \\
\leq K\left[\int_{[0,1]}\left(\left(t_{1}^{j}-u_{1}\right)_{+}^{H_{1}\left(t^{j}\right)-\frac{1}{2}}-\rho_{1, k}^{j}\left(u_{1}\right)\right)^{2} d u_{1}\right]^{\frac{1}{2}}
\end{gathered}
$$

where we have used the Hölder inequality and Lemma 2.1.

It follows from (2.20) and (2.25) that when $k \rightarrow \infty$,

$$
\mathbb{E}\left[\left|\int_{[0,1]^{d}}\left[\left(t_{1}^{j}-u_{1}\right)_{+}^{H_{1}\left(t^{j}\right)-\frac{1}{2}}-\rho_{1, k}^{j}\left(u_{1}\right)\right]\left[\prod_{i=2}^{d}\left(t_{i}^{j}-u_{i}\right)_{+}^{H_{i}\left(t^{j}\right)-\frac{1}{2}}\right] \theta_{n}(u) d u\right|\right] \stackrel{U}{\rightarrow} 0
$$

where $\stackrel{U}{\rightarrow}$ denotes uniform convergence with respect to $n$.

Repeating the above procedure, we can get

$$
\mathbb{E}\left|X_{n}\left(t^{j}\right)-X_{n}^{j, k}\right| \stackrel{U}{\rightarrow} 0, \text { as } k \rightarrow \infty .
$$


Therefore,

$$
I_{1} \stackrel{U}{\rightarrow} 0, \text { as } k \rightarrow \infty
$$

Similarly, by the mean value theorem, we can get

$$
I_{2} \leq K \max _{1 \leq j \leq q}\left\{\mathbb{E}\left|X\left(t^{j}\right)-X^{j, k}\right|\right\}
$$

Applying the Hölder inequality and the properties of the stochastic integral, we can get

$$
\mathbb{E}\left|X\left(t^{j}\right)-X^{j, k}\right| \leq K\left[\int_{[0,1]^{d}}\left(\prod_{i=1}^{d} \rho_{i, k}^{j}\left(u_{i}\right)-\prod_{i=1}^{d}\left(t_{i}^{j}-u_{i}\right)_{+}^{H_{i}\left(t^{j}\right)-\frac{1}{2}}\right)^{2} d u\right]^{\frac{1}{2}} .
$$

Using the same method as the proof of (2.27), we can get that

$$
I_{2} \stackrel{U}{\rightarrow} 0, \text { as } k \rightarrow \infty \text {. }
$$

Due to the result established by Wichura [17, the law of the process

$$
\int_{0}^{t_{1}} \cdots \int_{0}^{t_{d}} \theta_{n}(u) d u
$$

converges weakly to the law of a $d$-parameter Wiener process. Then, the linear combinations of increments of

$$
\int_{0}^{t_{1}} \cdots \int_{0}^{t_{d}} \theta_{n}(u) d u
$$

will converge in law to the same combinations of increments of the $d$-parameter Wiener process. So

$$
I_{3} \rightarrow 0
$$

as $n \rightarrow \infty$.

Combining (2.17), (2.22), (2.27), (2.30) and (2.31), we can get that Theorem 2.1 holds.

Acknowledgment The author would like to thank an anonymous referee for the insightful suggestions and helpful comments. This work was supported by Natural Science Foundation of Guangxi, China (2012GXNSFBA053010).

\section{References}

[1] Ayache, A., Léger. S. (2000). Fractional and Multifractional Brownian sheet. Preprint.

[2] Barnes, J. A., Allan, D. W. (1966). A statistical model of flicker noise. Proc. IEEE. $54,176-178$.

[3] Bardina, X., Florit, C. (2005). Approximation in law to the d-parameter fractional Brownian sheet based on the funcitonal invariance principle. Rev. Mat. Iberoamericana 21, 1037-1052. 
[4] Bardina, X., Jolis, M., Tudor, C. A. (2003). Weak convergence to the fractional Brownian sheet and other two-parameter Gaussian processes. Stat. Prob. Lett. 65, $317-329$.

[5] Bickel, P.J., Wichura, M. J. (1971). Convergence criteria for multiparameter stochastic processes and some applications. The annals of Mathematical statistics $42,1656-1670$.

[6] Ciesielski, I., Kamont, A. (1995). Lévy's fractional Brownian random field and function spaces. Acta. Sci. Math. (szeged). 60, 99-118.

[7] Dai, H., Li, Y. (2011). A note on approximiation to mulitifractional Brownian motion. Science China Mathematics 54 (10), 2145-2155

[8] Dai, H. (2012). Weak convergence to multifractional Brownian motion of RiemannLiouville type in Besov spaces. Journal of Applied Mathematics and Computing $38,601-615$.

[9] Herbin, E. (2006). From N parameter fractional Brownian motions to N parameter multifractional Brownian motions. Rocky Mount. J. Math. 36, 1249-1284.

[10] Khoshnevisan, D.(2002). Multiparameter processes, An introduction to random fields. Springer, New York.

[11] Kamont, A. (1996). On the fractional anisotropic Wiener field. Probab. Math. Statist. 16, 85-98.

[12] Mandelbrot, B., Van Ness, J. W. (1968). Fractional Brownian motions, fractional noises and applications. SIAM. Review 10, 422-437.

[13] Meerschaert, M., Wu, D., Xiao, Y. (2008). Local times of multifractional Brownian sheets. Bernoulli 14, 865-898.

[14] Lim, S. C. (2001). Fractional Brownian motion and multifractional Brownian motion of Riemann-Liouville type. J. Phys. A: Math. Gen. 34, 1301-1310.

[15] Lim, S.C., Muniandy, S. V. (2000). On some possible generalizations of fractional Brownian motion. Phys. Lett. A. 266, 140-145.

[16] Lim, S.C., Muniandy, S. V. (2002). Self-similar Gaussian processes for modeling anomalous diffusion. Phys. Rev. E. 66, 021114/1-021114/14.

[17] Wichura, M.J. (1969). Inequalities with applcations to the weak convergence of random processes with multi-dimensional time parameters. The annals of Mathematics Statistics 40, 681-687.

[18] Wu, D., Xiao, Y. (2007). Geometric properties of fractional Brownian sheets. J. Fourier. Anal. Appl. 13, 1-37. 